\title{
The Measurement of Capital Stock of China
}

\author{
Shide Feng ${ }^{1,2, a}$ \\ ${ }^{1}$ School of Economics (Faculty), Faculty of Political Science, Lomonosov Moscow State University, Lenin mountain No.1 \\ ${ }^{2}$ Country Faculty of Political Science, Moscow state institute of international relations, Ave. Vernadskogo, 76, Moscow, Russia \\ Federation
}

\begin{abstract}
Capital stock is an important indicator in economics, it is an important indicator to measure the social and economic development status. But the research of capital stock is not wildly contributed. This paper is to measure capital stock and forecast future capital stock with time series models. The author conducts empirical methods based on the measurement methods of previous scholars. The paper concludes that the capital stock is growing stably, and with established new mathematical models and time series models, and combined with the latest data, measures the capital stock of China.
\end{abstract}

\section{Introduction}

Capital stock is an important indicator in economics, especially for economists who focus on economic growth. There is a lot of classical models containing the capital stock. for example, but up to now, there is no Sophisticated and generally-accepted standard accounting method. The capital stock is an important indicator to measure the social and economic development status.

The research of capital stock is not wildly contributed. The data is hard to get, this research gap will take a lot of time of scholars to measure the stock during their work. There are no recent data for provincial capital stock, and the data is usually stop 2018, because the statistics bureau did not provide new data. This paper is to measure capital stock for the recent 24 years' capital stock of 31 provinces.

In this paper, the capital stock data of 31 provinces on the mainland was remeasured the author's models. At the same time, the paper makes a forecast for the next 5 years until 2022. these data will benefit other scholars whose research contains the capital stock elements.

\section{Critical Literature Review}

Chen (2012) measures the depreciation rate and capital stock [1]. Chen uses a new econometric model to estimate the depreciation rate, uses four models, uses the maximum likelihood estimation method, and uses the Monte Carlo method to ensuring accuracy at the same time. The depreciation rate is related to the economic growth rate. At the same time, China's economic structure is constantly changing with economic reforms, and the capital stock is also constantly changing. This article refers to its measurement model section to measure the depreciation rate, and the conclusions about the depreciation rate and economic growth rate are applied to the base period stock estimate.

Liu (2016) measures the state-own capital stock, Capital stock and capital services are the basis for measuring the total amount of capital [2]. Therefore, this paper measure the scale and accounting of China's stateowned fixed capital stock, and adopts the perpetual inventory method and Harrod-Domar model to provide important data support and methods for the subsequent research of China's state-owned capital. Provide the data and methods supports for scholars.

Based on the calculation of the depreciation rate, the depreciation of fixed capital in various regions is theoretically derived [3]. With these outcomes, Li (2016) adopts the depreciation of fixed capital in various regions from 1993 to 2012, and calculates the depreciation of fixed capital in China from 1993 to 2012. The capital coefficient is slowly increasing, which further validates the previous research. Using the depreciation of China 's fixed capital, the estimated depreciation rate of China 's fixed capital during 19922012 was about $5 \%$. Based on the estimated fixed capital depreciation rate, the capital stock of China from 1993 to 2012 is calculated, and its value is between the capital stock estimated by previous research.

Zhang and Cao (2019) studied the capital stock data and measured economic data using the perpetual inventory method [4]. At the same time, it compares the data of other authors and provides a reference benchmark for the conclusion of the article.

ashidefeng508@gmail.com 


\section{3 mthodology}

The paper adopts a different capital perpetual inventory method compared the previous research, the model comes from Solow Growth Model.

$$
\begin{gathered}
K_{t}=I+(1-\delta) K_{t-1} \\
I=s Y
\end{gathered}
$$

Combine (2) and (3). We have function (4).

$$
\begin{aligned}
K_{t} & =s Y+(1-\delta) K_{t-1} \\
h & =s \cdot(1-o-j)
\end{aligned}
$$

The $\mathrm{h}$ is the adjusted saving rate, $\mathrm{o}$ is the rate of the population who are under 15 years old, $\mathrm{j}$ o is the rate of the population who are over 65 years old, these parts of people are more intended to consume rather than saving.

$$
K_{t}=h Y+(1-\delta) K_{t-1}
$$

About the uncomplete investment data, we use averaged $\mathrm{h}$ (past 2 years and future 2 years) to measure.

$$
I=Y \cdot h
$$

About the year of benchmark.

$$
\begin{array}{r}
I=k_{t}-(1-\delta) k_{t-1} \\
K_{t}=I_{t+1}-\delta K_{t}
\end{array}
$$$$
q=\frac{K_{t}}{Y_{t}}
$$$$
K_{t}=\frac{I_{t+1}-\Delta K}{\delta_{t}}=\frac{I_{t+1}}{\delta_{t}+\frac{\Delta K}{K_{t}}}
$$

The capital stock growth rate is similar with the economic growth rate.

$$
K_{t}=\frac{I_{t+1}}{\delta_{t}+G R_{t}}
$$

GR is the economic growth rate.

$$
K_{0}=\frac{I_{1}}{\delta_{0}+G R_{0}}
$$

Thus, the first periods capital original reserve can be measured.

\begin{tabular}{|c|c|}
\hline & Coefficient \\
\hline$\alpha$ & 0.40 \\
\hline Intercpt & 0.7 \\
\hline$\delta_{1}$ & 0.058 \\
\hline$\delta_{2}$ & -0.02 \\
\hline
\end{tabular}

About the depreciation rate.

$$
\begin{gathered}
=\sum_{i=0}^{t-1} I_{t-1}\left(1-\delta_{t}\right)+\left(1-\delta_{t}\right) K_{0} \\
Y=A K^{\alpha} L^{1-\alpha} \\
\ln (Y)=\ln (A)+\alpha \ln (L)+(1-\alpha) \ln (k) \\
\delta_{t}=\delta_{1}+\delta_{2} x_{2 t}
\end{gathered}
$$

According to Chen, the depreciation express as above, can be shown as above.

TABLE I. THE OUTCOME OF EMPIRTICAL STUDY
After measurement of capital stocks, adopt ARIMA model to analyze the future quantities. The all result will be illustrated in chapter IV.

\section{Rseults}

TABLE II. THE CAPITITAL STOCK OF PROVINCES (100 MILLIONS)

\begin{tabular}{|c|c|c|c|c|}
\hline & BEIJING & TIANJING & HEBEI & SHANXI \\
\hline 1994 & 1838.907 & 716.675 & 2224.3 & 777.7369 \\
\hline 1995 & 2486.707 & 1060.694 & 3118.253 & 1031.496 \\
\hline 1996 & 2798.649 & 1409.545 & 4242.114 & 1345.686 \\
\hline 1997 & 3484.102 & 1821.602 & 5546.26 & 1692.105 \\
\hline 1998 & 4198.884 & 2239.545 & 6940.415 & 2145.321 \\
\hline 1999 & 4899.278 & 2654.502 & 8376.764 & 2471.652 \\
\hline 2000 & 5600.269 & 3126.893 & 9934.797 & 2817.938 \\
\hline 2001 & 7049.797 & 3721.998 & 11552.33 & 3353.801 \\
\hline 2002 & 8736.378 & 4397.546 & 13286.46 & 3974.852 \\
\hline 2003 & 10687.71 & 5264.132 & 15316.6 & 4828.287 \\
\hline 2004 & 13119.48 & 6414.092 & 18020.23 & 5986.458 \\
\hline 2005 & 15130.79 & 7931.905 & 21337.05 & 7330.321 \\
\hline 2006 & 17470.43 & 9614.159 & 25033.02 & 8865.983 \\
\hline 2007 & 20361.2 & 11561.66 & 29498.29 & 10940.13 \\
\hline 2008 & 23352.41 & 14267.85 & 34842.51 & 13583.44 \\
\hline 2009 & 26506.38 & 17199.56 & 40460.52 & 15861.91 \\
\hline 2010 & 30225.24 & 20825.05 & 47286.25 & 18959.63 \\
\hline 2011 & 34180.25 & 25190.81 & 55652.42 & 22822.15 \\
\hline 2012 & 38266.46 & 30063.11 & 64035.37 & 26660.82 \\
\hline 2013 & 42634.97 & 35307.73 & 72590.27 & 30223.63 \\
\hline 2014 & 46727.13 & 40985.31 & 80851.71 & 33476.57 \\
\hline 2015 & 50850.33 & 46119.78 & 88321.49 & 35989.46 \\
\hline 2016 & 56030.42 & 51333.57 & 95982.62 & 38314.82 \\
\hline 2017 & 61823.52 & 56607.93 & 103287.2 & 41312.38 \\
\hline 2018 & 67616.62 & 61932.47 & 110385.2 & 44621.03 \\
\hline 2019 & 73409.72 & 67296.75 & 117363.5 & 47929.67 \\
\hline 2020 & 79202.83 & 72693.3 & 124272.5 & 51238.32 \\
\hline 2021 & 84995.93 & 78115.68 & 131141.4 & 54546.97 \\
\hline 2022 & 90789.03 & 83558.91 & 137986.9 & 57855.61 \\
\hline
\end{tabular}


TABLE III. THE CAPITITAL STOCK OF PROVINCES (100 MILLIONS)

\begin{tabular}{|c|c|c|c|c|}
\hline & $\begin{array}{l}\text { I } \\
\text { MOGNOLIA }\end{array}$ & LIAONING & JILIN & $\begin{array}{l}\text { HEILONG } \\
\text { JIANG }\end{array}$ \\
\hline 1994 & 599.9531 & 3201.848 & 729.3189 & 1081.83 \\
\hline 1995 & 815.4261 & 3969.137 & 1007.723 & 1603.76 \\
\hline 1996 & 1035.681 & 4805.956 & 1338.031 & 2369.76 \\
\hline 1997 & 1306.064 & 5744.817 & 1650.716 & 3103.45 \\
\hline 1998 & 1617.31 & 6734.582 & 2002.93 & 3833.78 \\
\hline 1999 & 1925.143 & 7748.185 & 2378.178 & 4502.4 \\
\hline 2000 & 2269.665 & 8900.071 & 2742.931 & 5305.4 \\
\hline 2001 & 2720.625 & 10111.38 & 3206.239 & 6006.53 \\
\hline 2002 & 3206.15 & 11413.02 & 3739.655 & 6735.99 \\
\hline 2003 & 3919.656 & 13033.99 & 4316.111 & 7633.49 \\
\hline 2004 & 4935.347 & 14919.2 & 5064.345 & 8749.71 \\
\hline 2005 & 6340.197 & 17380.65 & 6145.114 & 10511.9 \\
\hline 2006 & 8174.719 & 20435.69 & 7490.324 & 12490.2 \\
\hline 2007 & 10663.05 & 24395.66 & 9215.995 & 14638.1 \\
\hline 2008 & 14198.29 & 29414.62 & 11396.17 & 17069 \\
\hline 2009 & 17980.36 & 34806.65 & 13862.83 & 19201.5 \\
\hline 2010 & 22645.95 & 41524.81 & 16992.7 & 22095.7 \\
\hline 2011 & 28366.46 & 49981.51 & 21014.33 & 25669.9 \\
\hline 2012 & 34360.77 & 59072.74 & 25405.78 & 29402.7 \\
\hline 2013 & 40281.62 & 68370.37 & 29923.52 & 32982.7 \\
\hline 2014 & 46369.75 & 77343.68 & 34819.38 & 36087.1 \\
\hline 2015 & 51996.92 & 85183.18 & 39463.47 & 38941.6 \\
\hline 2016 & 56891.73 & 87587.84 & 44324.53 & 41413.3 \\
\hline 2017 & 59761.31 & 90183.82 & 48878.07 & 43694.8 \\
\hline 2018 & 60902.66 & 92779.81 & 53421.28 & 45895.3 \\
\hline 2019 & 60569.22 & 95375.79 & 57828.83 & 48061.2 \\
\hline 2020 & 58977.27 & 97971.77 & 62186.23 & 50212.5 \\
\hline 2021 & 56311.36 & 100567.8 & 66468.43 & 52357.4 \\
\hline 2022 & 52728.98 & 103163.7 & 70703.73 & 54499.8 \\
\hline
\end{tabular}

TABLE IV. THE CAPITITAL STOCK OF PROVINCES (100 MILLIONS)

\begin{tabular}{|r|r|r|r|r|}
\hline & SHANGHAI & JIANGSU & ZHEJIANG & \multicolumn{1}{c|}{ ANHUI } \\
\hline 1994 & 2266.375 & 4065.224 & 2401.491 & 826.6986 \\
\hline 1995 & 3157.202 & 5864.498 & 3726.934 & 1203.114 \\
\hline 1996 & 4112.249 & 7842.31 & 5046.602 & 1929.74 \\
\hline 1997 & 5302.724 & 9964.981 & 6678.231 & 2524.166 \\
\hline 1998 & 6596.873 & 12273.94 & 8338.787 & 3142.055 \\
\hline 1999 & 7952.282 & 14698.11 & 10057.73 & 3682.408 \\
\hline 2000 & 9468.402 & 17370.48 & 11874.54 & 4223.908 \\
\hline 2001 & 11291.01 & 20135.58 & 13850.15 & 4770.65 \\
\hline 2002 & 13153.96 & 23232.89 & 16205.74 & 5398.893 \\
\hline 2003 & 15435.72 & 27084.97 & 19333.12 & 6093.354 \\
\hline 2004 & 18424.96 & 31792.6 & 23405.94 & 7057.696 \\
\hline 2005 & 21195.64 & 38298.9 & 27453.57 & 8280.427 \\
\hline 2006 & 24350.92 & 45780.43 & 32194.46 & 9715.894 \\
\hline 2007 & 28188.6 & 54693.87 & 38107.08 & 11540.47 \\
\hline 2008 & 32239.53 & 65353.54 & 44994.31 & 13770.14 \\
\hline 2009 & 36483.22 & 77018.94 & 52099.02 & 16514.3 \\
\hline 2010 & 41051.26 & 91389.63 & 61217.58 & 20038.79 \\
\hline
\end{tabular}

\begin{tabular}{|r|r|r|r|r|}
\hline 2011 & 45798.87 & 108015.2 & 71625.32 & 24491.14 \\
\hline 2012 & 50307.52 & 125647.9 & 82169.03 & 29430.12 \\
\hline 2013 & 55117.22 & 143748.7 & 93524 & 35077.84 \\
\hline 2014 & 59770.44 & 161263.4 & 104371.8 & 40839.55 \\
\hline 2015 & 64564.75 & 178286.9 & 115425.9 & 46522.02 \\
\hline 2016 & 70258.37 & 196405.6 & 127802.9 & 52419.14 \\
\hline 2017 & 76395.06 & 216895 & 140343.1 & 58878.06 \\
\hline 2018 & 82563.43 & 239318.5 & 153299.7 & 65794.85 \\
\hline 2019 & 88731.81 & 263320 & 166652.1 & 73084.81 \\
\hline 2020 & 94900.18 & 288608.8 & 180380.4 & 80678.91 \\
\hline 2021 & 101068.6 & 314947.9 & 194465.7 & 88520.89 \\
\hline 2022 & 107236.9 & 342143.8 & 208890.2 & 96564.91 \\
\hline
\end{tabular}

TABLE V. THE CAPITITAL STOCK OF PROVINCES (100 MILLIONS)

\begin{tabular}{|r|r|r|r|r|}
\hline & FUJIAN & JIANGXI & SHANDONG & HENAN \\
\hline 1994 & 848.3136 & 618.7282 & 3025.103 & 1721.948 \\
\hline 1995 & 1398.962 & 835.5369 & 4557.576 & 2541.622 \\
\hline 1996 & 2085.426 & 1192.534 & 6382.923 & 3553.271 \\
\hline 1997 & 2879.072 & 1580.808 & 8386.037 & 4649.127 \\
\hline 1998 & 3795.824 & 1997.438 & 10483.18 & 5820.931 \\
\hline 1999 & 4756.783 & 2422.75 & 12652.53 & 7005.969 \\
\hline 2000 & 5790.643 & 2789.406 & 15154.05 & 8248.529 \\
\hline 2001 & 6802.977 & 3199.557 & 17706.86 & 9475.913 \\
\hline 2002 & 7881.461 & 3722.653 & 20587.58 & 10775.79 \\
\hline 2003 & 9127.929 & 4435.732 & 24135.89 & 12250.92 \\
\hline 2004 & 10643.75 & 5288.396 & 28951.02 & 14345.83 \\
\hline 2005 & 12452.26 & 6308.85 & 35252.22 & 17307.64 \\
\hline 2006 & 14556.94 & 7650.626 & 42663.74 & 20829.12 \\
\hline 2007 & 17387.75 & 9319.559 & 51269.5 & 25649.12 \\
\hline 2008 & 20676.57 & 11409.29 & 61589.67 & 31679.6 \\
\hline 2009 & 24699.29 & 13701.57 & 73414.28 & 37656.57 \\
\hline 2010 & 29858.58 & 16497.89 & 87118.86 & 44749.84 \\
\hline 2011 & 35947.74 & 19928.89 & 102527.2 & 53090.23 \\
\hline 2012 & 42915.48 & 23608.76 & 118796.5 & 61724.88 \\
\hline 2013 & 50738.21 & 27523.32 & 136271.6 & 70357.65 \\
\hline 2014 & 58898.86 & 32069.54 & 154453.7 & 79160.06 \\
\hline 2015 & 67025.85 & 36125.28 & 172633 & 87626.13 \\
\hline 2016 & 75873.04 & 40492.67 & 188426 & 96460.16 \\
\hline 2017 & 85918.35 & 45204.82 & 203521.5 & 105867.8 \\
\hline 2018 & 97045.6 & 49633.93 & 218082.3 & 115634.9 \\
\hline 2019 & 109149.9 & 54358.05 & 232233.1 & 125627.4 \\
\hline 2020 & 122136.5 & 59235.19 & 246069.6 & 135761.2 \\
\hline 2021 & 135919.8 & 63975.42 & 259665.1 & 145983.4 \\
\hline 2022 & 150422.7 & 68951.37 & 273075.9 & 156261.2 \\
\hline & & & & \\
\hline
\end{tabular}

TABLE VI. THE CAPITITAL STOCK OF PROVINCES (100 MILLIONS)

\begin{tabular}{|l|r|r|r|r|}
\hline & \multicolumn{1}{|c|}{ HUBEI } & \multicolumn{1}{|c|}{ HUNAN } & $\begin{array}{l}\text { GUANG } \\
\text { DONG }\end{array}$ & GUANGXI \\
\hline 1994 & 1140.264 & 924.9485 & 4715.446 & 556.9698 \\
\hline 1995 & 1596.26 & 1368.51 & 6124.73 & 820.2908 \\
\hline 1996 & 2427.17 & 1880.31 & 7505.707 & 1202.766 \\
\hline 1997 & 3441.904 & 2489.395 & 9149.137 & 1593.171 \\
\hline
\end{tabular}




\begin{tabular}{|r|r|r|r|r|}
\hline 1998 & 4453.775 & 3148.773 & 10946.34 & 1894.59 \\
\hline 1999 & 5490.003 & 3756.022 & 12938.81 & 2197.299 \\
\hline 2000 & 6646.726 & 4473.412 & 15098.43 & 2488.551 \\
\hline 2001 & 7298.833 & 5146.381 & 18458.27 & 2830.141 \\
\hline 2002 & 8009.314 & 5870.181 & 21933.52 & 3253.004 \\
\hline 2003 & 8831.33 & 6850.196 & 26318.98 & 3766.718 \\
\hline 2004 & 9854.522 & 8144.694 & 32116.17 & 4471.498 \\
\hline 2005 & 11491.37 & 9543.782 & 38434.19 & 5272.392 \\
\hline 2006 & 13356.13 & 11282.74 & 46475.5 & 6333.347 \\
\hline 2007 & 15910.63 & 13677.76 & 56312.75 & 7747.02 \\
\hline 2008 & 19169.43 & 16805.59 & 67749.4 & 9535.467 \\
\hline 2009 & 23167.44 & 20513.78 & 79998.65 & 11391 \\
\hline 2010 & 28429.87 & 25473.38 & 94463.92 & 14021.25 \\
\hline 2011 & 34973.62 & 31563.72 & 110284.3 & 17448.62 \\
\hline 2012 & 42349.46 & 38431.1 & 125624.1 & 21041.41 \\
\hline 2013 & 50172.64 & 45854.39 & 141790.4 & 24761.22 \\
\hline 2014 & 58431.46 & 53693.18 & 160281.2 & 28517.22 \\
\hline 2015 & 66841.17 & 60753.19 & 178737.7 & 32478.63 \\
\hline 2016 & 75845.35 & 68262.43 & 199463.5 & 36535.31 \\
\hline 2017 & 85057.55 & 75431.61 & 222307.4 & 40071.18 \\
\hline 2018 & 94438.06 & 82690.36 & 246461.3 & 43287.64 \\
\hline 2019 & 103954.7 & 89827.59 & 271425.5 & 46308.21 \\
\hline 2020 & 113581.6 & 96965.48 & 296890.7 & 49208.64 \\
\hline 2021 & 123297.6 & 104049.9 & 322665.8 & 52035.38 \\
\hline 2022 & 133085.7 & 111117.5 & 348632.6 & 54816.93 \\
\hline
\end{tabular}

TABLE VII. THE CAPITITAL STOCK OF PROVINCES (100 MILLIONS)

\begin{tabular}{|r|r|r|r|r|}
\hline & HAINAN & CHONGQING & SICHUAN & GUIZHOU \\
\hline 1994 & 338.5799 & 671.704 & 527.0835 & 285.4371 \\
\hline 1995 & 428.1644 & 927.1505 & 670.8957 & 351.3644 \\
\hline 1996 & 515.962 & 1180.728 & 1410.842 & 372.1962 \\
\hline 1997 & 608.7761 & 1478.362 & 2204.957 & 445.211 \\
\hline 1998 & 706.3939 & 1786.644 & 3060.551 & 524.9209 \\
\hline 1999 & 808.1047 & 2075.172 & 3905.107 & 619.8773 \\
\hline 2000 & 919.4052 & 2380.28 & 4729.253 & 731.1904 \\
\hline 2001 & 1043.344 & 2744.638 & 5607.271 & 890.5565 \\
\hline 2002 & 1186.309 & 3147.993 & 6617.163 & 1078.519 \\
\hline 2003 & 1355.586 & 3562.449 & 7827.639 & 1345.288 \\
\hline 2004 & 1553.229 & 4136.029 & 9132.74 & 1649.612 \\
\hline 2005 & 1768.177 & 5068.056 & 10734.58 & 1807.167 \\
\hline 2006 & 2022.253 & 6091.824 & 12869.84 & 2043.474 \\
\hline 2007 & 2333.157 & 7300.179 & 15673.74 & 2429.211 \\
\hline 2008 & 2720.227 & 8452.632 & 19126.54 & 3171.831 \\
\hline 2009 & 3185.671 & 10375.52 & 23057.66 & 3900.806 \\
\hline 2010 & 3831.247 & 12771.6 & 27997.22 & 4839.726 \\
\hline 2011 & 4617.985 & 15893.38 & 34150.7 & 6120.698 \\
\hline 2012 & 5448.796 & 19336.68 & 40795.76 & 7789.528 \\
\hline 2013 & 6324.98 & 23106.66 & 47837.19 & 9817.821 \\
\hline 2014 & 7267.247 & 27220.45 & 55286.3 & 12008.58 \\
\hline 2015 & 7935.639 & 31583.65 & 62416.32 & 14470.92 \\
\hline 2016 & 8643.107 & 36363.9 & 70152.49 & 17167.51 \\
\hline 2017 & 9408.017 & 41399.42 & 78547.07 & 20270.76 \\
\hline 2018 & 10172.93 & 46727.89 & 87447.16 & 23690.91 \\
\hline 2019 & 10937.84 & 52282.96 & 96735.37 & 27358.03 \\
\hline 2020 & 11702.75 & 58060.16 & 106321.5 & 31217.62 \\
\hline
\end{tabular}

\begin{tabular}{|l|r|r|r|r|}
\hline 2021 & 12467.66 & 64025.4 & 116136.5 & 35227.2 \\
\hline 2022 & 13232.57 & 70164.69 & 126127.1 & 39353.68 \\
\hline
\end{tabular}

TABLE VIII. THE CAPITITAL STOCK OF PROVINCES (100 MILLIONS)

\begin{tabular}{|l|l|l|l|l|}
\hline TABL & & & & \\
& YUNAN & TIBET & SHANXI & GANSU \\
\hline 1994 & 894.6017 & 28.55579 & 616.0704 & 338.9347 \\
\hline 1995 & 1194.005 & 38.71065 & 820.2293 & 441.3241 \\
\hline 1996 & 1541.989 & 50.34965 & 1092.412 & 561.0226 \\
\hline 1997 & 1900.566 & 65.90091 & 1326.885 & 708.6972 \\
\hline 1998 & 2262.618 & 85.66882 & 1577.642 & 900.4719 \\
\hline 1999 & 2541.148 & 110.2398 & 1887.656 & 1113.079 \\
\hline 2000 & 2724.763 & 137.5414 & 2267.975 & 1330.472 \\
\hline 2001 & 3066.253 & 173.4907 & 2849.108 & 1573.972 \\
\hline 2002 & 3442.201 & 208.9636 & 3515.435 & 1879.849 \\
\hline 2003 & 3929.777 & 212.4208 & 4345.256 & 2243.842 \\
\hline 2004 & 4538.004 & 223.337 & 5437.09 & 2671.808 \\
\hline 2005 & 5094.321 & 255.8383 & 6783.475 & 3038.382 \\
\hline 2006 & 5786.832 & 343.1998 & 8522.798 & 3513.519 \\
\hline 2007 & 6786.796 & 396.725 & 10504.2 & 4102.282 \\
\hline 2008 & 8375.208 & 471.3951 & 13138.35 & 4771.756 \\
\hline 2009 & 9662.479 & 544.0434 & 15666.64 & 5458.301 \\
\hline 2010 & 11286.82 & 645.8367 & 19077.66 & 6421.506 \\
\hline 2011 & 13322.9 & 780.3174 & 23391.94 & 7606.851 \\
\hline 2012 & 15501.31 & 916.9672 & 28216.29 & 8929.09 \\
\hline 2013 & 17915.85 & 1076.427 & 33547 & 10436.32 \\
\hline 2014 & 20310.79 & 1250.009 & 39145.16 & 11946.27 \\
\hline 2015 & 22674.88 & 1330.611 & 44391.78 & 13082.45 \\
\hline 2016 & 25255.43 & 1436.397 & 49825.39 & 14159.37 \\
\hline 2017 & 28171.28 & 1548.805 & 56301.47 & 15056.54 \\
\hline 2018 & 31087.14 & 1661.213 & 63397.29 & 15871.71 \\
\hline 2019 & 34003 & 1773.621 & 70861.55 & 16649.48 \\
\hline 2020 & 36918.86 & 1886.03 & 78544.84 & 17410.18 \\
\hline 2021 & 39834.72 & 1998.438 & 86358.35 & 18163.1 \\
\hline 2022 & 42750.58 & 2110.846 & 94249.28 & 18912.47 \\
\hline & & & & \\
\hline
\end{tabular}

TABLE X. THE CAPITITAL STOCK OF PROVINCES (100 MILLIONS)

\begin{tabular}{|r|r|r|r|r|}
\hline & QINGHAI & NINGXIA & XINIJINAG & \\
\hline 1994 & 101.4988 & 75.44193 & 485.471 & \\
\hline 1995 & 133.3783 & 111.8227 & 672.5178 & \\
\hline 1996 & 161.6134 & 151.6054 & 870.6068 & \\
\hline 1997 & 199.6279 & 196.6673 & 1090.928 & \\
\hline 1998 & 243.4022 & 243.746 & 1333.134 & \\
\hline 1999 & 289.2717 & 289.732 & 1552.529 & \\
\hline 2000 & 339.4236 & 327.8698 & 1789.535 & \\
\hline 2001 & 391.8861 & 388.6555 & 2135.358 & \\
\hline 2002 & 454.0116 & 455.1194 & 2486.421 & \\
\hline 2003 & 528.6393 & 546.4427 & 2963.679 & \\
\hline 2004 & 615.669 & 666.3147 & 3548.056 & \\
\hline 2005 & 711.8535 & 786.2229 & 4308.635 & \\
\hline 2006 & 834.2191 & 935.4726 & 5129.593 & \\
\hline
\end{tabular}




\begin{tabular}{|l|r|r|r|r|}
\hline 2007 & 995.8739 & 1162.53 & 6003.874 & \\
\hline 2008 & 1249.664 & 1509.466 & 7084.834 & \\
\hline 2009 & 1522.265 & 1939.684 & 8175.183 & \\
\hline 2010 & 1908.829 & 2473.955 & 9633.287 & \\
\hline 2011 & 2394.976 & 3122.64 & 11373.27 & \\
\hline 2012 & 2922.664 & 3799.189 & 13134.68 & \\
\hline 2013 & 3569.517 & 4508.429 & 15210.63 & \\
\hline 2014 & 4218.259 & 5201.935 & 17427.97 & \\
\hline 2015 & 4676.213 & 5798.834 & 19098.11 & \\
\hline 2016 & 5075.436 & 6427.549 & 20532.02 & \\
\hline 2017 & 5397.652 & 7032.714 & 21984.71 & \\
\hline 2018 & 5683.081 & 7622.884 & 23419.09 & \\
\hline 2019 & 5950.937 & 8203.503 & 24853.47 & \\
\hline 2020 & 6210.399 & 8778.041 & 26287.86 & \\
\hline 2021 & 6465.852 & 9348.706 & 27722.24 & \\
\hline 2022 & 6719.389 & 9916.905 & 29156.62 & \\
\hline
\end{tabular}

\section{Conclusion}

The data illustrates that the Chinese capital stock is stably growing, here are the capital stocks, and with forecast.

\section{Reference}

1. C. Chen, "Estimation of variable depreciation rate and mearsurement of capital stock," Journal of Economics and Research, vol. 12, pp. 72-85, 2014.

2. C. Liu, "Research on Estimation of China's Stateowned Fixed Capital Stock," 2016.

3. Z. Li , "Estimation of variable depreciation rate and mearsurement of capital stock," Journal of Economics Research Guide, vol. 30, pp. 104-106, 2016.

4. C.Zhang and T. Cao, "Measurement of capital stock (K)," Journal of Finance Research, Vol 10, pp. 6376, 2019. 